\title{
Serpins, New Therapeutic Targets for Hemophilia
}

\author{
Karen Aymonnier ${ }^{1,2, *}$ Charlotte Kawecki ${ }^{1,3, *}$ Véronique Arocas ${ }^{1,2}$ Yacine Boulaftali ${ }^{1,2}$ \\ Marie Christine Bouton ${ }^{1,2}$
}

${ }^{1}$ INSERM U1148-LVTS, Université de Paris, Paris, France
${ }^{2}$ CHU Xavier Bichat, Paris, France
${ }^{3}$ INSERM U1176-HITh, Université Paris-Sud (Université Paris-Saclay),
Le Kremlin-Bicêtre, France

Thromb Haemost 2021;121:261-269.

\author{
Address for correspondence Marie-Christine Bouton, PhD, INSERM \\ U1148-LVTS, Université de Paris, Paris, France \\ (e-mail: marie-christine.bouton@inserm.fr).
}

Yacine Boulaftali, PhD, INSERM U1148-LVTS, Université de Paris, Paris, France (e-mail: yacine.boulaftali@inserm.fr).

\begin{abstract}
Keywords

- coagulation inhibitors

- hemophilia A/B

- Serpins

Hemostasis is a tightly regulated process characterized by a finely tuned balance between procoagulant and anticoagulant systems. Among inherited hemostatic conditions, hemophilia is one of the most well-known bleeding disorders. Hemophilia A (HA) and B (HB) are due to deficiencies in coagulation factor VIII (FVIII) or FIX, respectively, leading to unwanted bleeding. Until recently, hemophilia treatment has consisted of prophylactic replacement therapy using plasma-derived or recombinant FVIII in cases of HA or FIX in cases of HB. Because FVIII and FIX deficiencies lead to an imbalance between procoagulant and anticoagulant systems, a recent upcoming strategy implies blocking of endogenous anticoagulant proteins to compensate for the procoagulant factor deficit, thus restoring hemostatic equilibrium. Important physiological proteins of the anticoagulant pathways belong to the serpin (serine protease inhibitor) family and, recently, different experimental and clinical studies have demonstrated that targeting natural serpins could decrease bleeding in hemophilia. Here, we aim to review the different, recent studies demonstrating that blocking serpins such as antithrombin, protein Z-dependent protease inhibitor, and protease nexin-1 or modifying a serpin like $\alpha 1$-antitrypsin could rebalance coagulation in hemophilia. Furthermore, we underline the potential therapeutic use of serpins for the treatment of hemophilia.
\end{abstract}

\section{Hemophilia}

Hemostasis is a highly complex, integrated, dynamic system that limits blood leakage after an injury. Coagulation results in the formation of a solid clot made of fibrin and platelet aggregates. It involves a cascade of amplifying enzymatic reactions initiated by the exposure of the extravascular protein tissue factor (TF) to blood, allowing the formation of the TFfactor VIIa (FVIIa) complex (-Fig. 1). This complex is able to activate small amounts of FIXa and FXa. Small quantities of FXa promote the generation of thrombin that amplifies its own production by inducing a positive feedback loop via activation of FXI and of the cofactors FV and FVIII. Thrombin is the key

* K.A. and C.K. contributed equally to this article. final effector of the coagulation cascade; it generates fibrin and

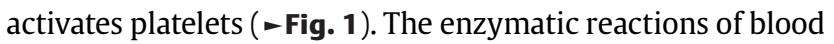
coagulation are mainly localized on platelet surfaces and are regulated by a set of physiological inhibitors, in particular, the serpins. In hemophilia, the delicate balance between pro- and anticoagulant proteins is disrupted, therefore resulting in abnormal bleeding (-Fig. 2). Indeed, hemophilia A (HA) and $B(H B)$ are monogenic X-linked recessive blood-clotting disorders due to deficiencies in the procoagulant factors FVIII or FIX, respectively, and characterized by repeated episodes of serious bleeding, primarily into their joints, that can lead to disability and pain. The main treatment for hemophilia consists of prophylactic replacement therapy using intravenous infusions of plasma-derived or recombinant FVIII in cases of

(C) 2020. Thieme. All rights reserved. Georg Thieme Verlag KG,

Rüdigerstraße 14 ,

70469 Stuttgart, Germany
DOI https://doi.org/ 10.1055/s-0040-1716751. ISSN 0340-6245. received

February 14, 2020

accepted after revision

August 7, 2020

published online

September 28, 2020 


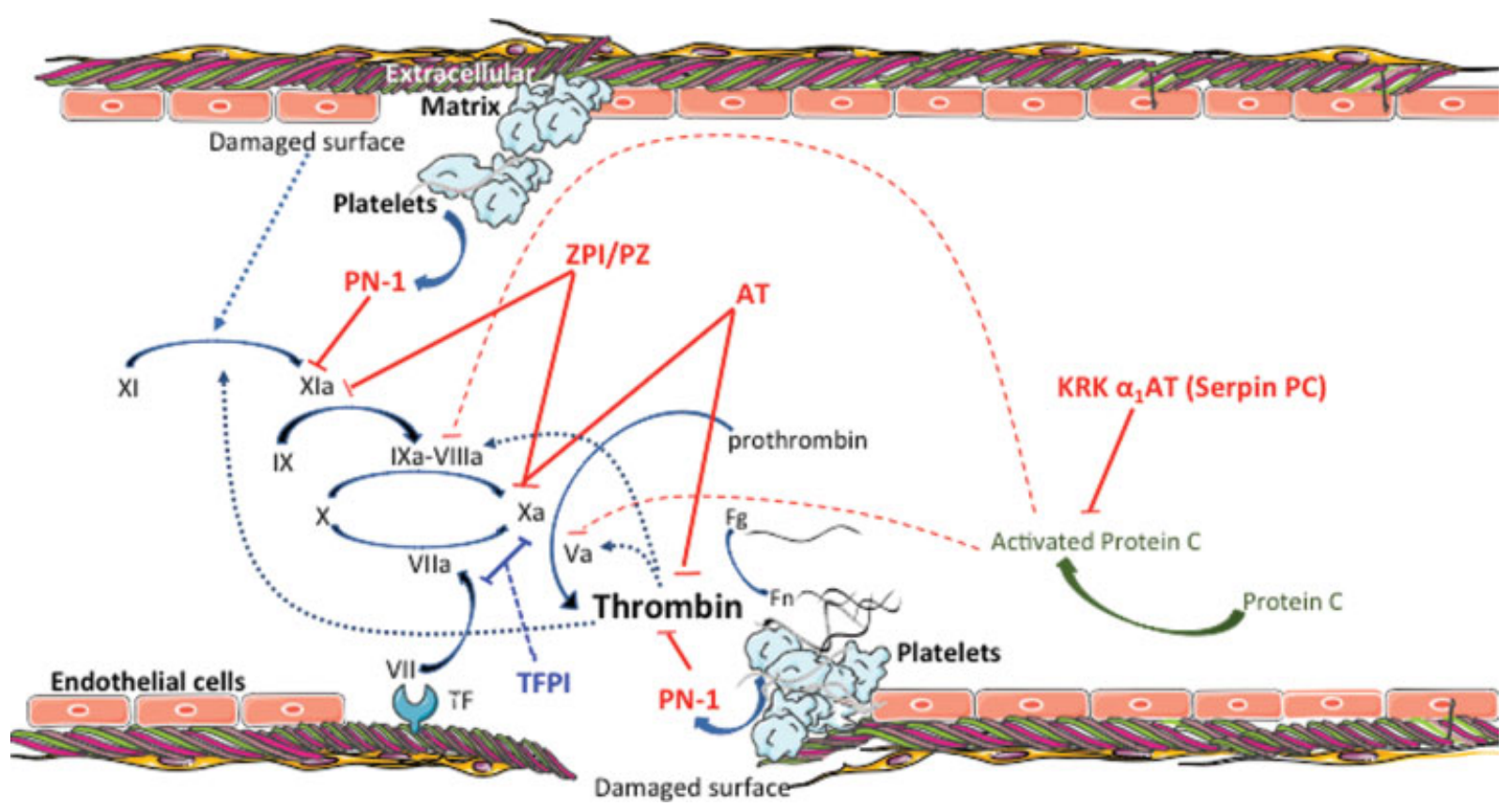

Fig. 1 Serpin targets to influence hemostasis in hemophilia. Schematic drawing of the blood coagulation cascade following a vascular injury and initiated by the exposure of tissue factor (TF). Thrombin, the key final effector of the coagulation cascade, generates fibrin (Fn) from plasma fibrinogen ( $F g$ ). Serpins (in red) from plasma, or released by activated platelets, regulate clot formation at different stages of the blood coagulation cascade.

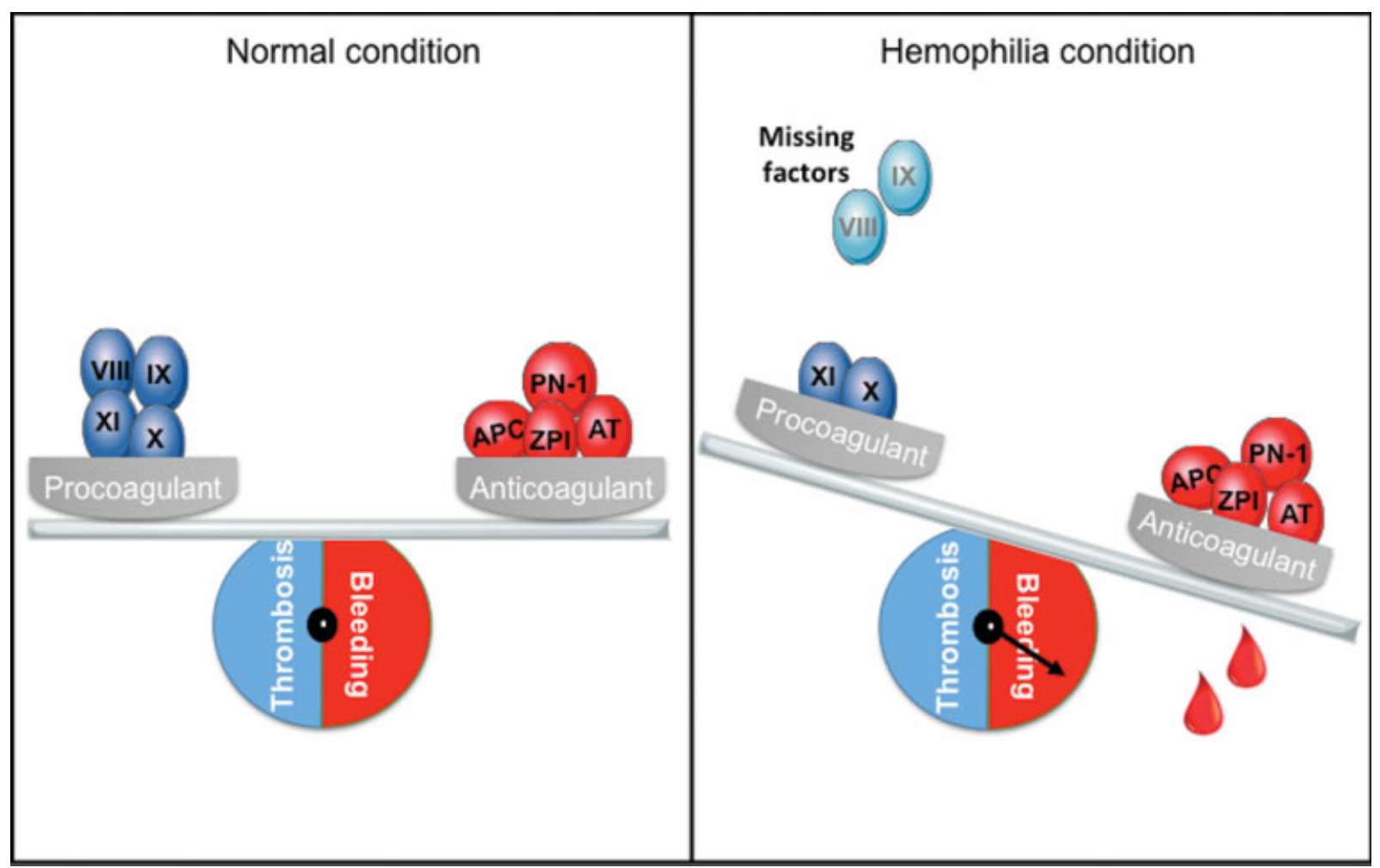

Fig. 2 Hemostatic imbalance in hemophilia. The deficiency in factor VIII (FVIII) or FIX leads to a disequilibrium of the finely tuned balance between procoagulant and anticoagulant proteins: activated protein C (APC); antithrombin (AT); protein Z-dependent protease inhibitor (ZPI); protease nexin-1 (PN-1).

HA or FIX in cases of HB. Despite remarkable improvements, challenges still remain in hemophilia treatment, in particular because of the need of frequent intravenous injections of FVIII or FIX and the development in some patients of neutralizing antibodies. These alloantibodies remain the most serious complication of replacement therapy because patients who develop inhibitors become refractory to the treatment. In such situations, bypassing agents are used to stop acute bleeds, but with limited success. ${ }^{1}$

In this context, other therapeutic approaches are now being developed or explored. They include other replacement therapies such as FVIII/FIX products, with an extended half-life, 
and gene therapy, or alternative therapies like the monoclonal FVIII-mimicking bispecific antibody, and agents targeting endogenous anticoagulant proteins including TF pathway inhibitor (TFPI), activated protein C (APC), or antithrombin $(\mathrm{AT}){ }^{2}$ The main strength of targeting natural inhibitors of coagulation is related to its application to both patients with $\mathrm{HA}$ and $\mathrm{HB}$, unlike the other strategies. Moreover, this nonfactor therapy can be used for both patients with and without inhibitors and should not lead to the development of inhibitors since it avoids or largely reduces factor infusions. Furthermore, these therapeutic molecules can be administered subcutaneously, a much less invasive route than the repeated intravenous injection of FVIII or FIX. Recently, different experimental studies have demonstrated that targeting two other natural anticoagulant serpins, namely protein $\mathrm{Z}$-dependent protease inhibitor (ZPI) and protease nexin-1 (PN-1), can also decrease bleeding in hemophilia. This review focuses on the different, recent strategies developed to block or modify serpins and features their potential therapeutic use for treatment of hemophilia (-Table $\mathbf{1}$ ).

\section{Serpins}

Serpins, a name derived from SERine Protease INhibitor, comprise a superfamily of proteins, widely distributed among organisms, which share a conserved tertiary structure and have evolved primarily to control the activity of serine and cysteine proteases. All serpin structures determined so far have the same basic fold composed of three major $\beta$-sheets, seven to nine $\alpha$-helices, and an exposed reactive center loop (RCL) that contains the primary recognition site for attacking proteases. ${ }^{3}$ The majority of the members of the serpin family function as protease inhibitors in a wide range of physiological processes, including blood coagulation and fibrinolysis. ${ }^{4}$ Indeed, the coagulation cascade and the protein $\mathrm{C}$ anticoagulant and fibrinolytic pathways are dominated by serine proteases regulated by antiproteases predominantly belonging to the serpin superfamily. Most serpins are suicide inhibitors. Unlike the classical lock-and-key type inhibitors, serpins use conformational change to present recognition sites contained in their exposed RCL that is cleaved by the target protease ( $\mathbf{F i g . ~ 3 ) . ~ I n ~}$ most cases, this cleavage leads to the formation of a serpinprotease complex covalently bound, in which the protease active site becomes inactivated via conformational deformation, and the serpin undergoes a massive structural change with the cleaved RCL inserted into the serpin body. By combining interactions of both an exposed RCL and exosites outside this loop with the active site and complementary exosites on the target protease, serpins can achieve remarkable specificity. ${ }^{5}$ Moreover, the activity of several serpins is modulated by glycosaminoglycans serving as a bridge between serpins and proteases, potentiating the inhibition rate. ${ }^{6}$

\section{Antithrombin}

In hemophilia, coagulation is rapidly quenched by two principal protease inhibitors: TFPI and AT. Therefore, both anticoagulant proteins have been targeted for hemophilia. TFPI is not a serpin but a Kunitz-type proteinase inhibitor that inhibits the FVIIa-TF complex and $\mathrm{FXa}^{7}$ (-Fig. 1). Therapeutic agents targeting TFPI have been extensively reviewed elsewhere. ${ }^{8}$ AT is a 58-kDa glycoprotein encoded by the SERPINC1 gene. It is synthesized in the liver and circulates in blood. It inhibits a large panel of blood coagulation proteases but functions as a key natural anticoagulant protein because of its high ability to inactivate thrombin and $\mathrm{FXa}^{9}$ (-Fig. 1).

The importance of AT in the regulation of coagulation has been strengthened by investigations with human subjects. Indeed, congenital and acquired AT deficiencies are known to be associated with an increased risk of venous thromboembolism. ${ }^{10,11}$ Moreover, the coinheritance of hemophilia with a deficiency of AT is associated with a milder bleeding phenotype. ${ }^{12}$ A small cohort study of patients with severe FVIII or FIX deficiency has shown that reduced AT activity attenuates the clinical severity of patients. ${ }^{13}$ Another study showed that endogenous thrombin potential was increased in vitro in human FVIII-deficient plasma with reduced AT levels. ${ }^{14}$ The decrease in bleeding tendency achieved by lowering AT was confirmed in studies performed in mice. FVIII-deficient mice with a heterozygous AT deficiency display increased thrombin generation and less in vivo bleeding compared with FVIII-deficient mice with normal AT levels. ${ }^{15}$

Taken together, these observations have led to the development of a therapeutic gene-silencing strategy designed to lower levels of AT in patients with hemophilia. A small interfering ribonucleic acid (siRNA) named fitusiran (ALN-AT3, Sanofi Genzyme, Cambridge, Massachusetts, United States), given subcutaneously, has been developed to block the hepatic synthesis of AT. ${ }^{16}$ This siRNA against AT has been shown to increase thrombin generation in human $\mathrm{HA}$ and $\mathrm{HB}$ plasma. In a preclinical study, fitusiran has been shown to enhance thrombus formation in a microvessel laser injury model and to improve hemostasis in a saphenous vein-bleeding model after subcutaneous administration in hemophilic mice. Moreover, it improved thrombin generation in a nonhuman primate model of HA with anti-FVIII inhibitors. ${ }^{16}$ Recently, another novel ATtargeting approach has been developed. It is based on llamaderived single domain antibody fragments able to restore hemostasis in HA and HB mice by inhibiting AT, and was shown to be safe and only weakly immunogenic. ${ }^{17}$

Fitusiran has been evaluated in hemophilia patients with and without inhibitors in phase 1 and 2 clinical trials. In the phase 1 study, healthy volunteers and patients with HA or HB without inhibitory antibodies received three injections of fitusiran administered either once-weekly (at a dose varying from 0.015 to $0.075 \mathrm{mg} / \mathrm{kg}$ ) or once-monthly (either at a dose of 0.225 to $1.8 \mathrm{mg} / \mathrm{kg}$ or at a fixed $80 \mathrm{mg}$ dose). Phase 1 showed that once-monthly subcutaneous administration of fitusiran resulted in a stable, dose-dependent mean maximum reduction in AT of 70 to $89 \%$ from baseline and increased thrombin generation in patients with HA or HB without inhibitory alloantibodies. ${ }^{18}$ The trial was interrupted because of the death of a patient with HA who suffered fatal cerebral venous sinus thrombosis after the combination of fitusiran with repeated administration of FVIII. ${ }^{19-21}$ This suspension was subsequently lifted after additional investigator and patient 

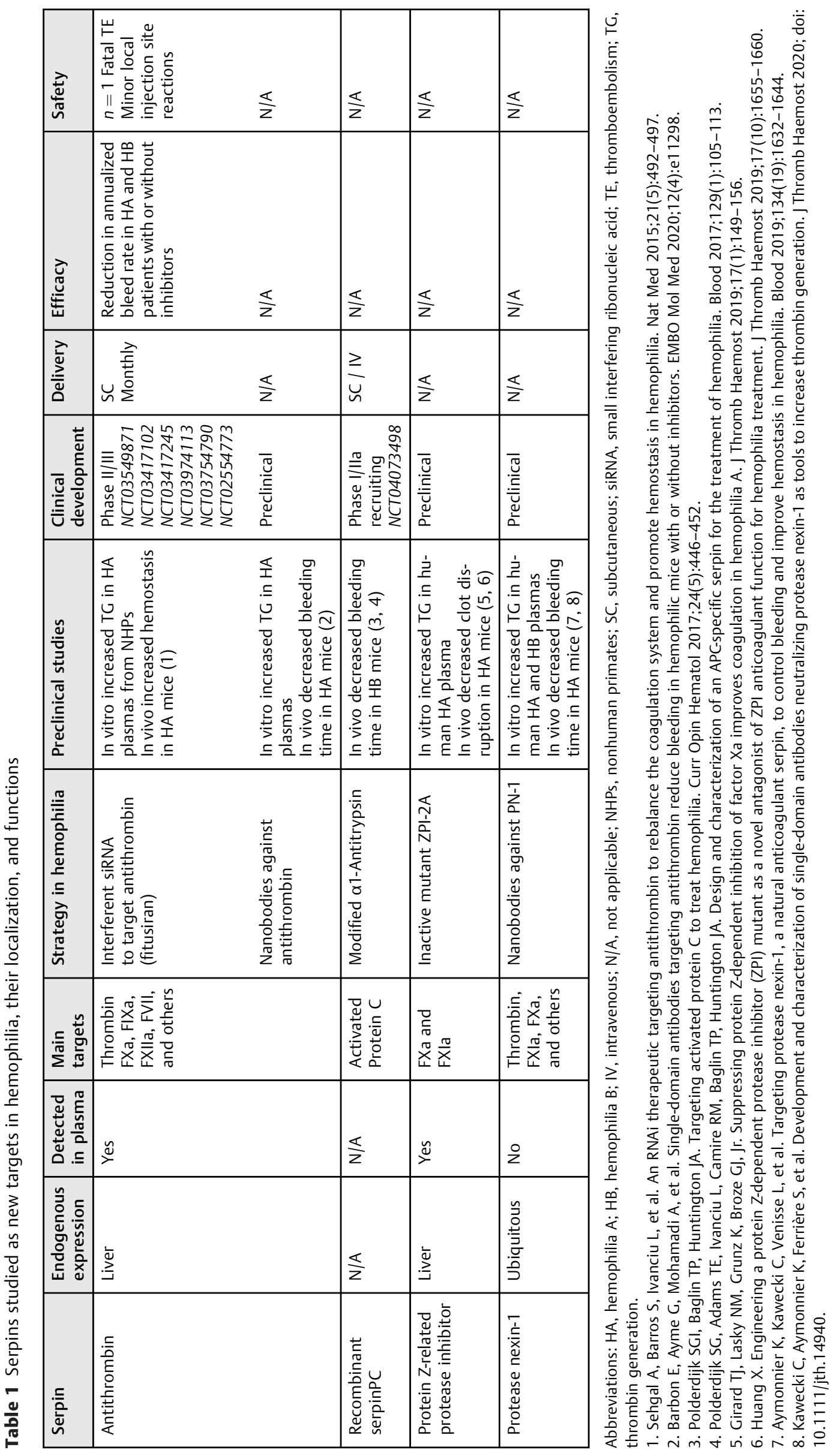

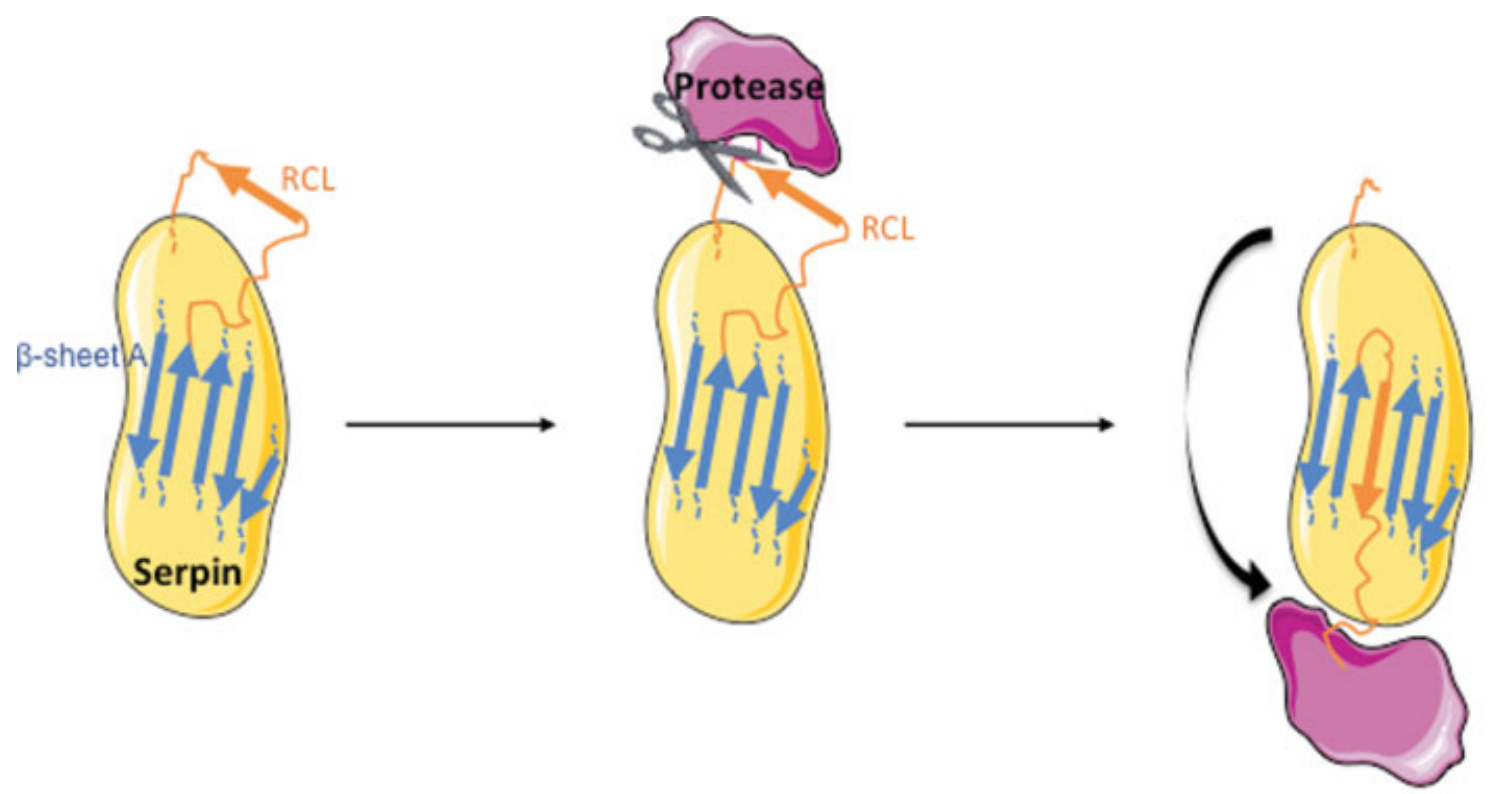

\section{Native serpin}

Michaelis complex

Final covalent complex

Fig. 3 The serpin inhibitory mechanism. The exposed serpin reactive center loop (RCL) is cleaved by the target protease and inserts into the $\beta$ sheet $A$ resulting in a covalent complex in which both serpin and protease are inactivated.

education strategies and the revision of the bleeding management guidelines. Since the introduction of that protocol, there have been no related thrombotic events. ${ }^{22}$ The interim analysis of the phase 2 extension study with patients with HA or HB (including 14 with inhibitors), receiving monthly fixed subcutaneous doses of 50 or $80 \mathrm{mg}$ fitusiran showed significant declines in annualized bleeding rate. Patients treated with both dosages experienced a reduction of nearly $80 \%$ in AT levels. Such a drop reduced the overall median annualized bleeding rate to 1 , with $48 \%$ of patients remaining bleed-free throughout the study. No antibodies against fitusiran developed in any patients, and breakthrough bleeds were successfully treated with replacement factors or bypassing agents according to the revised guidelines for reduced doses. ${ }^{23}$ Oncemonthly subcutaneous administration of $80 \mathrm{mg}$ fitusiran is currently under evaluation in phase 3 clinical trials.

Fitusiran has the advantage of being easily administered, since it is injected subcutaneously. However, as already outlined by the clinical trials, targeting AT will require caution.

\section{SerpinPC}

Another strategy consisted of developing a serpin inhibiting APC. Indeed, APC is a powerful anticoagulant that limits thrombin production by proteolytic degradation of factors FVa and FVIIIa, essential cofactors of the coagulation cascade (-Fig. 1). An analysis of the existing literature on the coinheritance of hemophilia with the FV Leiden mutation, a mutation making FV resistant to inactivation by APC, suggested that FV Leiden may compensate for the low FVIII or IX levels, resulting in increased thrombin generation and an ensuing attenuation of clinical symptoms. ${ }^{24}$ This conclu- sion was reinforced by an in vitro study showing that the combination of HA and FV Leiden led to an increase in plasma thrombin formation ${ }^{25}$ and by in vivo studies showing that FV Leiden improves hemostasis in murine hemophilia models. ${ }^{26}$ Therefore, APC inhibition was hypothesized to restore the hemostatic balance in hemophilia. However, no single natural inhibitor has high specificity for this protease. Specific synthetic APC inhibitors have been shown to increase thrombin generation in severe hemophilia ${ }^{27}$ but such APC inhibitors would require concentrations too high to potentially compensate for severe hemophilia in vivo. It has also been proposed to target protein $S$ because it serves as an essential cofactor for both TFPI and APC. This approach has indeed been reported to rebalance coagulation and prevent hemarthrosis in murine models of hemophilia. ${ }^{28}$ Another strategy to inhibit APC was described by Polderdijk et al who used a mutant form of SERPINA1 or $\alpha 1$-antitrypsin ( $\alpha 1 \mathrm{AT})$, named $\alpha 1 \mathrm{AT}$ Pittsburg, as a serpin template to obtain a strong and specific inhibitor of APC. ${ }^{29}$ In contrast to the natural form of $\alpha 1 \mathrm{AT}, \alpha 1 \mathrm{AT}$ Pittsburg is a mutant serpin known to rapidly inhibit not only APC but also procoagulant proteases such as thrombin. ${ }^{30}$ To improve specificity for APC over other coagulation serine proteases, the investigators mutated the amino acid residues around the cleavage site of the RCL with Lys substitution creating a KRK $\alpha 1$ AT mutant. This engineered serpin, named serpinPC, ${ }^{31}$ was shown to be effective in increasing thrombin generation in vitro and, after injection in $\mathrm{HB}$ mice to restore fibrin and platelet deposition in an intravital laser injury model and to reduce blood loss in the tail clip assay. SerpinPC (ApcinteX Ltd, United Kingdom) has a potentially long half-life, can inhibit APC specifically and rapidly, and can be administered subcutaneously. 
SerpinPC is under evaluation in ongoing phase I/II clinical trials for healthy volunteers and patients with all types of hemophilia. ${ }^{32}$ These trials will provide information on the safety, tolerability, and efficacy of serpinPC in treating hemophilia. As a variant of a natural inhibitor, immunogenicity and consequent cross-reactivity might be a potential issue, even if the risk of immune tolerance is supposed to be low for proteins present at high concentration in plasma, as is the case for $\alpha 1-\mathrm{AT}$. Importantly, because APC also exerts essential cytoprotective and anti-inflammatory functions, these trials should indicate whether targeting APC with serpinPC will allow inhibition of its anticoagulant activity in absence of any associated inflammatory response.

\section{Protein Z (PZ)-Dependent Proteinase Inhibitor}

Recently, two studies have shown that blocking another anticoagulant serpin, protein Z(PZ)-ZPI, could enhance thrombin generation in human hemophilia plasma. ${ }^{33,34} \mathrm{ZPI}$ is a 72kDa glycoprotein encoded by the SERPINA10 gene. ${ }^{35}$ This natural serpin is synthesized by hepatic cells and circulates in the plasma, either freely or as a tight equimolar complex with its cofactor PZ. ${ }^{36}$ The ZPI/PZ complex antagonizes coagulation by rapidly inhibiting membrane-associated FXa in the presence of calcium and phospholipids as cofactors. ${ }^{37}$ In the absence of PZ, ZPI can also inhibit FXIa, a key factor in the amplification of the coagulation cascade that also contributes to blood clot formation ${ }^{37}$ (-Fig. 1). As observed with many other inhibitors of the serpin family, heparin accelerates both FXa and FXIa inhibitions by ZPI/PZ and ZPI, respectively. ${ }^{38,39}$ In contrast to AT or PC, whose modest deficiency is clearly associated with an increased risk of thrombosis in humans, no clear link was found between ZPI/PZ deficiency and thrombotic events in humans. ${ }^{40}$ Nevertheless, the importance of the $\mathrm{ZPI} / \mathrm{PZ}$ complex as a regulator of hemostasis has been evidenced by the following findings: (1) the ZPI/PZ complex delays in vitro thrombin formation and decreases the level of thrombin generation, ${ }^{41}$ (2) PZ influences the prothrombotic phenotype in FV Leiden patients, ${ }^{42}$ (3) in combination with the FV Leiden genotype, ZPI deficiency results in a severe thrombosis phenotype in mice, including intravascular coagulation and hemorrhage, ${ }^{43}$ and (4) the upregulation of the ZPI/PZ anticoagulation system has been reported to be correlated with an increased bleeding rate and increased joint bleeding of severe HA patients. ${ }^{44}$ Therefore, it was naturally hypothesized that neutralizing ZPI/PZ could improve hemostasis in hemophilia. A first study confirmed this hypothesis by demonstrating that ZPI or PZ deficiency reduced bleeding in a tail vein rebleeding assay and improved plasma thrombin generation in HA mice. ${ }^{33}$ Moreover, the authors of this study showed that an antibody blocking PZ interaction with ZPI enhanced coagulation in human HA plasma to a degree equivalent to that obtained with hemophilic plasma supplemented with around $15 \%$ FVIII activity. Unfortunately, this antibody could not represent a potential therapeutic tool since it has a low potency. The authors suggested that an agent designed to interfere with the binding of the ZPI/PZ complex with phospholipid surfaces might be a more potent inhibitor than the antibody used in their studies.
Another approach to neutralize ZPI/PZ anticoagulant activity was published recently by Huang et al, who chose to engineer an inactive ZPI double mutant named ZPI-2A. ${ }^{41} \mathrm{ZPI}-$ $2 \mathrm{~A}$ comprises one alanine mutation in the RCL to inactivate its anticoagulant function and a second alanine mutation in its binding site to $\mathrm{PZ}$ to enhance its affinity for this latter. Therefore, ZPI-2A could antagonize the anticoagulant function of ZPI and restore hemostasis in hemophilia. This was illustrated by the data obtained in thrombin generation assays in a purified system or in normal/hemophilia plasma samples, showing that ZPI/PZ activity could be reversed by ZPI-2A in a dose-dependent manner, with a threefold molar excess being sufficient to fully reverse the ZPI/PZ inhibition of thrombin generation. ZPI-2A should efficiently compete with and displace wild-type (WT) ZPI from its complex with $\mathrm{PZ}$ resulting in inactive $\mathrm{ZPI}-2 \mathrm{~A} / \mathrm{PZ}$ complexes and uncomplexed WT ZPI. It is noteworthy that the remaining uncomplexed WT ZPI is not totally devoid of anticoagulant activity since it can still inhibit FXIa, a factor that could contribute to hemostasis, especially in individuals with a bleeding tendency. ${ }^{45}$ Moreover, evaluating the impact of ZPI-2A administration in vivo in murine hemophilia models by monitoring, for example, its impact on blood loss after tail clip, remains to be performed.

As mentioned for serpinPC, there may also be some concerns regarding the immunogenic potential of such a variant of a natural inhibitor. Moreover, the PZ/ZPI complex, apart from its anticoagulant activity, may have an antiinflammatory role that would bring up the question of the safety of targeting ZPI/PZ. ${ }^{46}$

\section{Protease Nexin-1}

$\mathrm{PN}-1$ is the last serpin target proposed to treat bleeding in hemophilia. PN-1 is a 50-kDa glycoprotein encoded by the SERPINE2 gene. Unlike AT, $\alpha 1-\mathrm{AT}$, or ZPI, PN-1 is barely detectable in the circulating blood. Indeed, PN-1 can be considered as a cellular serpin since it is expressed by many cell types including vascular cells, blood cells, and, in particular, platelets ${ }^{47}$ ( - Fig. 1). Most platelet PN-1 is secreted during platelet activation. ${ }^{48}$ Although PN-1 can inhibit a broad spectrum of serine proteases, thrombin is its preferred target. PN-1 was shown in vitro to be the most effective inhibitor of thrombin, more effective than AT, even in the presence of heparin. ${ }^{47}$ It is also a more efficient inhibitor of FXIa than any other natural serpin inhibitor, which underlines the additional impact of $\mathrm{PN}-1$ on the coagulation cascade. ${ }^{49}$

In the past decade, platelet $\mathrm{PN}-1$ was an unrecognized endogenous negative regulator of thrombin generation. We demonstrated that platelets from PN-1-deficient mice display an increased sensitivity to thrombin and an enhanced procoagulant activity. ${ }^{48}$ Moreover, thrombus formation was accelerated and facilitated in PN-1-deficient mice after induction of vascular lesions. ${ }^{48}$ Taken together, these data resulted in the hypothesis that PN-1 blockade would allow not only increased thrombin activity but also increased thrombin generation in hemophilia. Indeed, using double 
Table 2 Serpins studied as new targets in hemophilia, advantages and limitations

\begin{tabular}{|c|c|c|c|c|}
\hline Approaches & $\begin{array}{l}\text { Antithrombin } \\
\text { targeting }\end{array}$ & $\begin{array}{l}\text { Recombinant } \\
\text { serpinPC }\end{array}$ & ZPI targeting & Protease nexin-1 targeting \\
\hline $\begin{array}{l}\text { Common } \\
\text { strengths }\end{array}$ & \multicolumn{4}{|c|}{$\begin{array}{l}\boldsymbol{V} \text { Can treat both HA and HB patients } \\
\boldsymbol{V} \text { Can treat patients with inhibitors } \\
\boldsymbol{V} \text { Can be administrated subcutaneously }\end{array}$} \\
\hline $\begin{array}{l}\text { Specific } \\
\text { advantages }\end{array}$ & $\begin{array}{l}\text { High hemostatic } \\
\text { properties }\end{array}$ & $\begin{array}{l}\text { High hemostatic } \\
\text { properties }\end{array}$ & Potential low thrombotic risk & Potential low thrombotic risk \\
\hline $\begin{array}{l}\text { Safety } \\
\text { concerns }\end{array}$ & $\begin{array}{l}\text { Management of } \\
\text { thrombotic risk }\end{array}$ & $\begin{array}{l}\text { - Inflammatory risk } \\
\text { - Reduction of } \\
\text { cytoprotective } \\
\text { functions }\end{array}$ & $\begin{array}{l}\text { - Weak procoagulant response } \\
\text { - Risk of immunogenicity }\end{array}$ & $\begin{array}{l}\text { - Weak procoagulant response } \\
\text { - Potential adverse cerebral } \\
\text { tissue impact }\end{array}$ \\
\hline
\end{tabular}

Abbreviations: HA, hemophilia A; HB, hemophilia B; ZPI, protein Z-related protease inhibitor.

knockout (KO) mice for PN-1 and FVIII, we showed that PN-1 deficiency could reduce bleeding in hemophilic mice. ${ }^{50}$ Moreover, we showed that double KO mice exhibited an increase in platelet recruitment and fibrin(ogen) deposition at the surface of injured vessels compared with hemophilic mice. Importantly, the rate and extent of thrombus formation remained much lower than those measured in WT mice, strongly suggesting that targeting PN-1 would not be linked to thrombotic risk in the hemophilia context. We also demonstrated that a PN-1-neutralizing antibody has no effect on thrombin generation in platelet-poor plasma but could significantly improve it in platelet-rich plasma from hemophilic patients. This data underlines the critical role of PN-1 expressed in platelets. It is now recognized that platelet-dependent factors, beyond the expression of phospholipids, are necessary for platelet-dependent thrombin generation. Therefore, PN-1 is one of these platelet-dependent factors that could play a regulatory role in hemophilia. Single domain antibodies, recently developed to bind specifically to PN-1 and neutralize its activity toward thrombin, could be explored for their potential therapeutic applications. $^{51}$

Unlike AT or ZPI, PN-1 does not circulate in the plasma, but is present in large amounts in platelets. However, one concern is the ubiquitous cellular expression of PN-1, in particular in the brain where it is thought to display a neuroprotective role, ${ }^{52}$ and which could lead to potential off-target effects of an agent directed against PN-1. Because the platelet surface plays a central role in the promotion and regulation of thrombin generation, targeting PN-1 specifically in platelets could be a means to reinforce thrombin generation on the platelet surface directly at the site of injury.

\section{Conclusion}

The ideal therapeutic agent for hemophilia treatment must be safe, well-tolerated, highly efficient in bleeding prevention for both types of hemophilia, without adverse effects (thrombotic risk) and should require administration at a low frequency, via a simple route. Targeting anticoagulant proteins such as serpins, but also like the anticoagulant TFPI, could fulfill many of these criteria. Moreover, these strategies are potentially suitable for patients with rare bleeding disorders characterized by insufficient thrombin generation, other than hemophilia. However, the main disadvantage of such strategies is the nonnegligible risk of adverse thrombotic events. This is inherent to strategies that target key negative regulators of coagulation, and is illustrated by the fitusiran trials that were first transiently halted, as well as by some clinical trials developed to evaluate high affinity antibodies to TFPI that were also interrupted or paused, because of safety issues (nonfatal thrombotic events). Therefore, it is important to amend trial protocols to better mitigate the risks, as in the fitusiran or TFPI trials. Since gene KO of both PN-1 and ZPI in mice produces viable individuals and does not lead to an obvious phenotype (in contrast to AT, TFPI, or PC deficiencies), the question is open as to whether targeting moderate (e.g., ZPI and PN-1) versus major (e.g., AT, APC pathway, TFPI) negative regulators of coagulation would be an advantage regarding the thrombotic risks. On the other hand, targeting moderately anticoagulant serpins may not be sufficient to produce the required procoagulant response. Targeting each of these anticoagulant serpins has thus its pros and cons (-Table 2). The ongoing clinical trials for fitusiran, serpinPC, or TFPI will provide answers to help to determine the ideal target, and clinical studies will also be needed to evaluate the potential beneficial hemostatic effect of targeting anticoagulant serpins like PN-1 and ZPI.

\section{Funding}

This work was supported by INSERM, Université de Paris, and grants from CSL Behring-SFH, the Agence Nationale de la Recherche (ANR-14-OHRI-0013), the Bayer Hemophilia Award Program, and the National Blood Foundation. K.A. was the recipient of a PhD fellowship from the Société Française d'Hématologie (SFH).

\section{Conflict of Interest}

M.-C.B. has a patent application related to $\mathrm{PN}-1$ targeting in hemophilia.

\section{Acknowledgments}

The authors thank Mary Osborne-Pellegrin for help in editing this manuscript as well as Emmanuelle de Raucourt and Yohann Repessé for their helpful discussions. 


\section{References}

1 Franchini M, Coppola A, Tagliaferri A, Lippi G. FEIBA versus NovoSeven in hemophilia patients with inhibitors. Semin Thromb Hemost 2013;39(07):772-778

2 Butterfield JSS, Hege KM, Herzog RW, Kaczmarek R. A molecular revolution in the treatment of hemophilia. Mol Ther 2020;28(04): 997-1015

3 Gettins PG, Olson ST. Exosite determinants of serpin specificity. J Biol Chem 2009;284(31):20441-20445

4 Rau JC, Beaulieu LM, Huntington JA, Church FC. Serpins in thrombosis, hemostasis and fibrinolysis. J Thromb Haemost 2007;5(Suppl 1):102-115

5 Olson ST, Gettins PG. Regulation of proteases by protein inhibitors of the serpin superfamily. Prog Mol Biol Transl Sci 2011;99:185-240

6 Huntington JA. Serpin structure, function and dysfunction. J Thromb Haemost 2011;9(Suppl 1):26-34

7 Peterson JA, Maroney SA, Mast AE. Targeting TFPI for hemophilia treatment. Thromb Res 2016;141(Suppl 2):S28-S30

8 Chowdary P. Anti-tissue factor pathway inhibitor(TFPI) therapy: a novel approach to the treatment of haemophilia. Int J Hematol 2020;111(01):42-50

9 Quinsey NS, Greedy AL, Bottomley SP, Whisstock JC, Pike RN. Antithrombin: in control of coagulation. Int J Biochem Cell Biol 2004;36(03):386-389

10 Marciniak E, Farley CH, DeSimone PA. Familial thrombosis due to antithrombin 3 deficiency. Blood 1974;43(02):219-231

11 Patnaik MM, Moll S. Inherited antithrombin deficiency: a review. Haemophilia 2008;14(06):1229-1239

12 Franchini M, Montagnana M, Targher G, et al. Interpatient phenotypic inconsistency in severe congenital hemophilia: a systematic review of the role of inherited thrombophilia. Semin Thromb Hemost 2009;35(03):307-312

13 Shetty S, Vora S, Kulkarni B, et al. Contribution of natural anticoagulant and fibrinolytic factors in modulating the clinical severity of haemophilia patients. Br J Haematol 2007;138(04):541-544

14 Szlam F, Taketomi T, Sheppard CA, Kempton CL, Levy JH, Tanaka KA. Antithrombin affects hemostatic response to recombinant activated factor VII in factor VIII deficient plasma. Anesth Analg 2008;106(03):719-724

15 Bolliger D, Szlam F, Suzuki N, Matsushita T, Tanaka KA. Heterozygous antithrombin deficiency improves in vivo haemostasis in factor VIIIdeficient mice. Thromb Haemost 2010;103(06):1233-1238

16 Sehgal A, Barros S, Ivanciu L, et al. An RNAi therapeutic targeting antithrombin to rebalance the coagulation system and promote hemostasis in hemophilia. Nat Med 2015;21(05):492-497

17 Barbon E, Ayme G, Mohamadi A, et al. Single-domain antibodies targeting antithrombin reduce bleeding in hemophilic mice with or without inhibitors. EMBO Mol Med 2020;12(04):e11298

18 Pasi KJ, Rangarajan S, Georgiev P, et al. Targeting of antithrombin in hemophilia A or B with RNAi therapy. N Engl J Med 2017;377 (09):819-828

19 World Federation of Hemophilia. Alnylam suspends fitusiran dosing due to thrombotic event in phase 2 open-label extension study. Available at: https://news.wfh.org/. Accessed March 6, 2018

20 Nogami K, Shima M. New therapies using nonfactor products for patients with hemophilia and inhibitors. Blood 2019;133(05): 399-406

21 Machin N, Ragni MV. An investigational RNAi therapeutic targeting antithrombin for the treatment of hemophilia A and B. J Blood Med 2018;9:135-140

22 World Federation of Hemophilia. Update: FDA lifts suspension of fitusiran trial. Available at: https://news.wfh.org/. Accessed March 6, 2018

23 Pasi KJ, Lissitchkov T, Georgiev P, et al. Fitusiran, an investigational RNAi therapeutic targeting antithrombin for the treatment of hemophilia: Interim results from a phase 2 extension study in patients with hemophilia A or B with and without inhibitors. Res Pract Thromb Haemost 2017;1(01):25-26
24 Franchini M, Lippi G. Factor V Leiden and hemophilia. Thromb Res 2010;125(02):119-123

25 van 't Veer C, Golden NJ, Kalafatis M, Simioni P, Bertina RM, Mann KG. An in vitro analysis of the combination of hemophilia A and factor V(LEIDEN). Blood 1997;90(08):3067-3072

26 Schlachterman A, Schuettrumpf J, Liu JH, et al. Factor V Leiden improves in vivo hemostasis in murine hemophilia models. J Thromb Haemost 2005;3(12):2730-2737

27 Butenas S, Orfeo T, Kalafatis M, Mann KG. Peptidomimetic inhibitors for activated protein C: implications for hemophilia management. J Thromb Haemost 2006;4(11):2411-2416

28 Prince R, Bologna L, Manetti M, et al. Targeting anticoagulant protein $S$ to improve hemostasis in hemophilia. Blood 2018;131 (12):1360-1371

29 Polderdijk SG, Adams TE, Ivanciu L, Camire RM, Baglin TP, Huntington JA. Design and characterization of an APC-specific serpin for the treatment of hemophilia. Blood 2017;129(01): 105-113

30 de Maat S, Sanrattana W, Mailer RK, et al. Design and characterization of $\alpha 1$-antitrypsin variants for treatment of contact systemdriven thromboinflammation. Blood 2019;134(19):1658-1669

31 Polderdijk SGI, Baglin TP, Huntington JA. Targeting activated protein C to treat hemophilia. Curr Opin Hematol 2017;24(05): $446-452$

32 Apcinte XLtd. has begun dosing in a Phase I/II clinical trial. Available at: http://www.apcintex.com/. Accessed August 29, 2020

33 Girard TJ, Lasky NM, Grunz K, Broze GJ Jr. Suppressing protein Zdependent inhibition of factor Xa improves coagulation in hemophilia A. J Thromb Haemost 2019;17(01):149-156

34 Huang X. Engineering a protein Z-dependent protease inhibitor (ZPI) mutant as a novel antagonist of ZPI anticoagulant function for hemophilia treatment. J Thromb Haemost 2019;17(10): $1655-1660$

35 Han X, Huang ZF, Fiehler R, Broze GJ Jr. The protein Z-dependent protease inhibitor is a serpin. Biochemistry 1999;38(34): 11073-11078

36 Tabatabai A, Fiehler R, Broze GJ Jr. Protein Z circulates in plasma in a complex with protein Z-dependent protease inhibitor. Thromb Haemost 2001;85(04):655-660

37 Han X, Fiehler R, Broze GJ Jr. Characterization of the protein Zdependent protease inhibitor. Blood 2000;96(09):3049-3055

38 Huang X, Rezaie AR, Broze GJ Jr, Olson ST. Heparin is a major activator of the anticoagulant serpin, protein Z-dependent protease inhibitor. J Biol Chem 2011;286(11):8740-8751

39 Yang L, Ding Q, Huang X, Olson ST, Rezaie AR. Characterization of the heparin-binding site of the protein z-dependent protease inhibitor. Biochemistry 2012;51(19):4078-4085

40 Corral J, González-Conejero R, Hernández-Espinosa D, Vicente V. Protein Z/Z-dependent protease inhibitor (PZ/ZPI) anticoagulant system and thrombosis. Br J Haematol 2007;137(02):99-108

41 Huang X, Swanson R, Kroh HK, Bock PE. Protein Z-dependent protease inhibitor (ZPI) is a physiologically significant inhibitor of prothrombinase function. J Biol Chem 2019;294(19):7644-7657

42 Kemkes-Matthes B, Nees M, Kühnel G, Matzdorff A, Matthes KJ. Protein $\mathrm{Z}$ influences the prothrombotic phenotype in Factor $\mathrm{V}$ Leiden patients. Thromb Res 2002;106(4-5):183-185

43 Zhang J, Tu Y, Lu L, Lasky N, Broze GJ Jr. Protein Z-dependent protease inhibitor deficiency produces a more severe murine phenotype than protein $\mathrm{Z}$ deficiency. Blood 2008;111(10): 4973-4978

44 Bolkun L, Galar M, Piszcz J, Lemancewicz D, Kloczko J. Plasma concentration of protein $\mathrm{Z}$ and protein $\mathrm{Z}$-dependent protease inhibitor in patients with haemophilia A. Thromb Res 2013;131 (03):e110-e113

45 Tillman BF, Gruber A, McCarty OJT, Gailani D. Plasma contact factors as therapeutic targets. Blood Rev 2018;32(06):433-448

46 Butschkau A, Nagel P, Grambow E, Zechner D, Broze GJ Jr, Vollmar B. Contribution of protein $\mathrm{Z}$ and protein $\mathrm{Z}$-dependent protease 
inhibitor in generalized Shwartzman reaction. Crit Care Med 2013;41(12):e447-e456

47 Arocas V, Bouton MC. Protease Nexin-1: a serpin involved in pathophysiology. In: Geiger M, Wahlmüller F, Furtmüller M, eds. The Serpin Family Proteins with Multiple Functions in Health and Disease. Switzerland: Springer International Publishing; 2015: 179-196

48 Boulaftali Y, Adam F, Venisse L, et al. Anticoagulant and antithrombotic properties of platelet protease nexin-1. Blood 2010;115 (01):97-106

49 Knauer DJ, Majumdar D, Fong PC, Knauer MF. SERPIN regulation of factor XIa. The novel observation that protease nexin 1 in the presence of heparin is a more potent inhibitor of factor XIa than C1 inhibitor. J Biol Chem 2000;275(48):37340-37346

50 Aymonnier K, Kawecki C, Venisse L, et al. Targeting protease nexin1 , a natural anticoagulant serpin, to control bleeding and improve hemostasis in hemophilia. Blood 2019;134(19):1632-1644

51 Kawecki C, Aymonnier K, Ferrière S, et al. Development and characterization of single-domain antibodies neutralizing protease nexin- 1 as tools to increase thrombin generation. J Thromb Haemost 2020. Doi: 10.1111/jth.14940

52 Monard D. SERPINE2/protease nexin-1 in vivo multiple functions: does the puzzle make sense? Semin Cell Dev Biol 2017; 62:160-169 